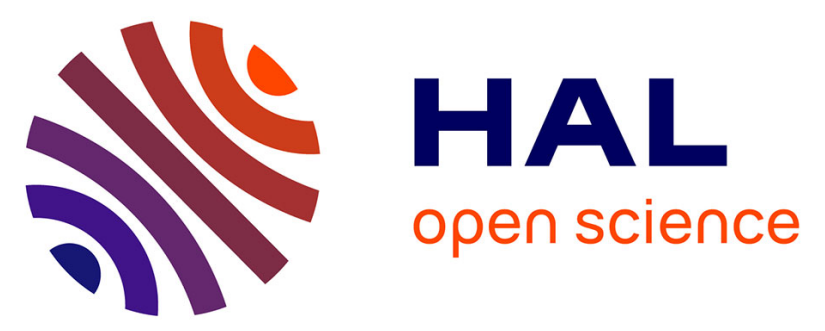

\title{
Sliding Mode Impedance Controlled Smart Fingered Microgripper for Automated Grasp and Release Tasks at the Microscale
}

\author{
Bilal Komati, Cédric Clévy, Philippe Lutz
}

\section{To cite this version:}

Bilal Komati, Cédric Clévy, Philippe Lutz. Sliding Mode Impedance Controlled Smart Fingered Microgripper for Automated Grasp and Release Tasks at the Microscale. 8th International Precision Assembly Seminar (IPAS), Jan 2018, Chamonix, France. pp.201-213, 10.1007/978-3-030-05931-6_18 . hal-02115835

\section{HAL Id: hal-02115835 \\ https://hal.inria.fr/hal-02115835}

Submitted on 30 Apr 2019

HAL is a multi-disciplinary open access archive for the deposit and dissemination of scientific research documents, whether they are published or not. The documents may come from teaching and research institutions in France or abroad, or from public or private research centers.
L'archive ouverte pluridisciplinaire HAL, est destinée au dépôt et à la diffusion de documents scientifiques de niveau recherche, publiés ou non, émanant des établissements d'enseignement et de recherche français ou étrangers, des laboratoires publics ou privés. 


\title{
Sliding Mode Impedance Controlled Smart Fingered Microgripper for Automated Grasp and Release Tasks at the Microscale
}

\author{
Bilal Komati ${ }^{1}$, Cédric Clévy ${ }^{1}$ and Philippe Lutz ${ }^{1}$ \\ ${ }^{1}$ FEMTO-ST Institute, Univ. Bourgogne Franche-Comté, CNRS, 24 rue Savary, F-25000 Be- \\ sançon, France
}

\begin{abstract}
The grasp and release of objects have been widely studied in robotics. At the microscale, this problem becomes more difficult due to the microscale specificities which are notably manifested by the high dynamics of microsystems, their small inertia, their fragility, the predominance of surface forces and the high complexity of integrating adapted sensors.

In this paper, the problem of the grasp/release task is considered at the microscale. A new nonlinear controller design based on Sliding Mode Impedance Control (SMIC) is proposed to automate the grasp/release of the micropart. The proposed controller controls dexterously the dynamic interaction between the microgripper and the micropart and forces the system to follow the desired dynamic relation (impedance). To perform the grasp/release task, a new smart-fingeredmicrogripper is designed. The microgripper is composed of an active finger with integrated force sensor and a passive finger.

The grasp/release of a micropart of size $50 \mu \mathrm{m} \times 350 \mu \mathrm{m} \times 2 \mathrm{~mm}$ is tested in experiments using the control scheme and the developed microgripper. The microgripper design and the control scheme tested show their effectiveness for the grasp/release at the microscale.
\end{abstract}

Keywords: Sliding Mode Impedance Control, microassembly, guiding task, Smart microgripper, piezoelectric actuator, force sensor.

\section{Introduction}

The use of robotic microassembly has increased in recent years due to the development of complex microsystems and the need for microassembly to develop more complex 3D microsystems [1], [2]. Moreover, the microassembly enables the integration of heterogeneous components issued from incompatible microfabrication processes.

The scaling effect has a strong influence because it exhibits, at the microscale, many problems that are not faced at the macroscale. The microscale specificities could be briefly summarized by the high dynamics of microsystems, their small inertia, their fragility, the predominance of surface forces and the necessity and complexity to integrate sensors notably to measure contact forces that have predominant effects and high signal to noise ratio sensors [3]. These facts increase the complexity of the manipulation 
especially when the interactions forces are not measured; thus, the importance of integrating force sensors into the microgripper to measure the interaction forces with high bandwidth.

The integration of force sensors into microgrippers has given rise to several works [4], [5], [6], [7], [8], [9], [10], [11]. Most of the works present monolithic fabricated microgrippers composed of an active finger and a force sensing finger. However, in these grippers, the actuated finger is independent from the force sensing finger and in this case the contact could not be detected between the actuated finger and the micropart, leading to the loss of the micropart and consequently an unsuccessful grasp of the micropart. Moreover, in the releasing task, the control of surface forces cannot be mastered because the measure of force is not done on the same actuated finger. For this reason, it is interesting to integrate the force sensor inside the same active finger of the microgripper. In this paper, a microgripper composed of a piezoelectric based active finger with an integrated piezoresistive force sensor and a passive finger is presented. The use of the piezoelectric actuator and piezoresistive force sensor enables not only having very small resolution for the actuation and force sensing but also having very high bandwidth for both actuation and control.

Another issue which can be faced in microassembly is the need for high dynamics in the control of interaction between the microgripper and the micropart to perform a stable grasp/release of the micropart. Impedance control presented by Hogan [12] has shown its significance at the macroscale to control the interaction between the robot and the environment and has been proven to have a good advantage for microscale applications in [13], [14]. However, the main limitations of impedance control is the force tracking capability and the ability to follow the desired impedance despite robot dynamics and parameter uncertainties. Some works have proposed performing parameter estimation in order to perform force tracking [15] and others proposed combining sliding mode control [16] with impedance control to force the system to follow the desired impedance of the system [17], [18]. Thus, the main contribution of this paper is the use of Sliding Mode Impedance Control (SMIC) with online parameter estimation technique in order to guarantee both the force tracking and the desired impedance of the system.

The paper is organized as follows. Section 2 presents the microgripper design. Section 3 develops the model of the microgripper. Section 4 presents the impedance control with force tracking scheme. Section 5 presents the experimental setup used. Section 6 presents the parameter identification of the system and the experimental investigations of the automated grasping task. Section 7 concludes the paper.

\section{Microgripper Design}

The microgripper is made of two fingers. One is called a smart finger composed of a piezoelectric actuator and piezoresistive force sensor. The second is a passive finger. The piezoelectric actuator presented in [4] is used. The piezoresistive force sensor has been presented in [19]. The fabrication of the complete gripper consists of assembling the force sensor on the tip of the piezoelectric actuator. Special care should be taken to 
perform the mechanical fixation of the actuator and the force sensor while separating the electrical signals. The dimensions, actuation and sensing ranges and resolutions will be detailed in Section 6. A scheme of the microgripper is shown in Fig. 1. The rigid part shown in Fig. 1 is used to help the integration of the force sensor into the microgripper and to facilitate the realization of the whole microgripper. In the proposed microgripper, the smart finger deforms until entering in contact with the micropart first, and then the micropart, pushed by the first finger, enters in contact with the second finger. Two models need to be developed for the microgripper in the cases of free motion (non-contact) and constrained motion (contact).

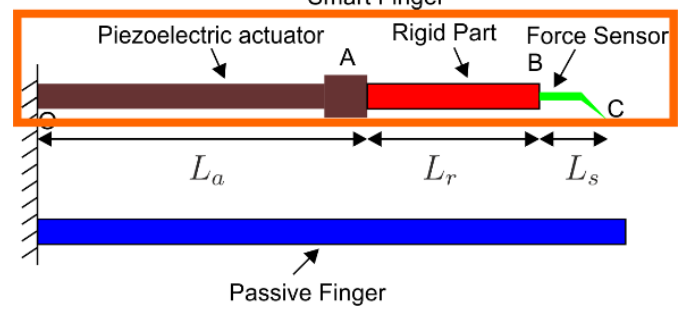

Fig. 1. Complete gripper scheme which is composed of two fingers: smart finger composed of a piezoelectric actuator with a piezoresistive force sensor; and passive finger.

\section{$3 \quad$ Microgripper Model}

In this section, the complete voltage/force/displacement model of the gripper is developed. The model is used to determine the control law and to estimate the position of the grippers end-effector.

\subsection{Model of the Passive Finger and of the Microcomponent}

The passive finger of the gripper is modeled as a mass-spring-damper system. The microcomponent to be manipulated is supposed to be rigid in the direction of the gripping and then it could be modeled as a mass. When a microcomponent is handled, the gripping force applied on the microcomponent by the gripper's smart finger, $F_{g}$, is given by the following:

$$
F_{g}=m_{p} \ddot{y}+b_{p}\left(\dot{y}-\dot{y}_{p}\right)+k_{p}\left(y-y_{p}\right)
$$

where $b_{p}$ and $k_{p}$ represents respectively the damping coefficient and the stiffness of the passive finger of the microgripper. $m_{p}$ is the mass of the microcomponent and of the passive finger $\left(m_{p}=m_{p_{1}}+m_{p_{2}}\right)$ where $m_{p_{1}}$ and $m_{p_{2}}$ are respectively the mass of the passive finger and of the micropart. $y$ is the current position of the microcomponent and $y_{p}$ is the position of the microcomponent just at contact (i.e. $f_{g}=0$ if $y<y_{p}$ ). 


\subsection{Model of the Smart Finger}

The smart finger of the microgripper is composed of a piezoelectric actuator (between points $\mathrm{O}$ and $\mathrm{A}$ in Fig. 1) where an end-effector is fixed on the actuator's tip A. The end-effector is composed of a rigid silicon part (between points A and B in Fig. 1) and a piezoresistive force sensor (between points $B$ and $C$ in Fig. 1). The setup and details of the actuator and force sensor are presented in section 5. The force sensor measures the force applied at the tip C. The sequence of the grasping task is shown in Fig. 2.

(a)

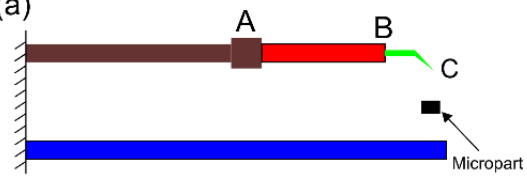

(b)

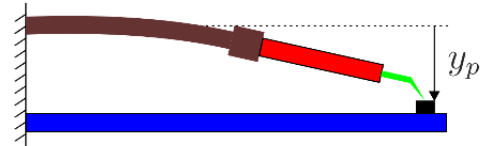

(c)

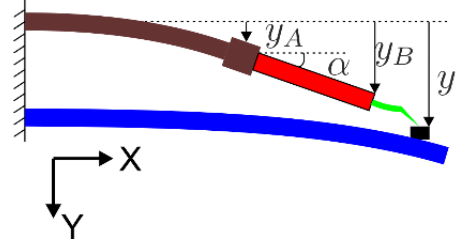

Fig. 2. Sequence of the microgripper during grasping: (a) without any applied voltage to the smart finger and no contact, (b) just at contact $(F=0)$ and (c) contact with $F \neq 0$.

The force sensor (between points B and C in Fig. 1) is modeled as a lever system with length $L_{s}$ (between points B and D of Fig. 3) with mass-spring-damper system (between points D and C of Fig. 3). Then the complete scheme of the grasping task is shown in Fig. 3.

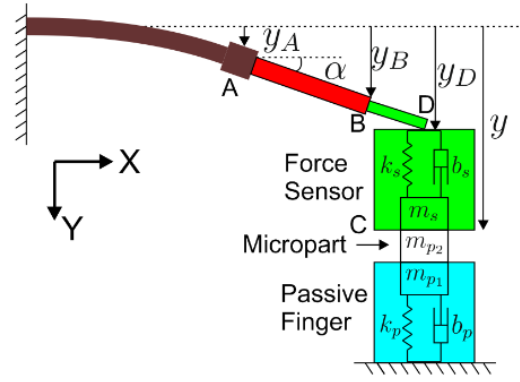

Fig. 3. Equivalent scheme of the complete gripper handling a microcomponent where the force sensor and the passive finger are modeled as mass-spring-damper systems.

The complete model of the smart finger is developed in [20] but will be briefly summarized in this section. The model of the piezoelectric actuator is developed using existing models in literature [21], [22], [23] as follows: 


$$
\left\{\begin{array}{c}
y_{A}(s)=\left[d_{p} U-s_{p} F_{A}-\frac{3}{2 L_{a}} s_{p} M_{A}\right] D(s)-\Gamma(s, U) \\
\alpha_{A}(s)=\left[\frac{2}{L_{a}} d_{p} U-\frac{3}{2 L_{a}} s_{p} F_{A}-\frac{3}{L_{a}^{2}} s_{p} M_{A}\right] D(s)-\frac{2}{L_{a}} \Gamma(s, U)
\end{array}\right.
$$

where $s$ is the Laplace variable, $y_{A}$ is the displacement of the actuator tip A and $\alpha_{A}$ is the angle at the actuator tip A, $d_{p}$ is the piezoelectric constant, $s_{p}$ is the elastic constant (it is the inverse of the stiffness), $L_{a}$ is the length of the actuator, $U$ is the applied voltage, $D(s)$ is a linear transfer function which models the dynamics of the actuator with a static gain of $1(D(0)=1)$ and $\Gamma(U, s)$ is an operator to represent the rate-dependent hysteresis of the actuator if we neglect the creep of the actuator.

As developed in [20], the rate-dependent hysteresis of the piezoelectric actuator can be modeled as a static hysteresis followed by the dynamics of the piezoelectric actuator, $D(s)$, as follows:

$$
\Gamma(u, s)=H_{s}(U) \cdot D(s)
$$

where $H_{S}(U)$ is a static hysteresis which does not depend on the dynamics of the actuator. It can be modeled, according to [23], [20], by the Bouc-Wen method in the time domain as follows:

$$
\dot{H}_{S}(U)=\lambda \dot{U}+\beta|\dot{U}| H_{S}(U)-\gamma \dot{U}\left|H_{S}(U)\right|
$$

where $\lambda$ is a parameter which determines the amplitude of the hysteresis and $\beta$ and $\gamma$ are parameters which determine the shape of the hysteresis.

According to literature, a piezoelectric actuator can be modeled as a mass-springdamper system with an active part that is a second order transfer function and is sufficient to model the dynamics of the actuator [20]. The transfer function $D(s)$ can be written as follows:

$$
D(s)=\frac{1}{a s^{2}+b s+1}
$$

In our work the force is not applied at the actuator tip A but at the force sensor $\mathrm{C}$ as shown in Fig. 2. A force applied on the force sensor tip $\mathrm{C}$ induces a reaction force and moment at the actuator tip A defined as follows:

$$
\left\{\begin{array}{c}
F_{A}=-F_{s}+m_{s} \ddot{y}_{C}=F_{g}+m_{s} \ddot{y}_{C} \\
M_{A}=-L F_{S}=L F_{g}
\end{array}\right.
$$

where $F_{g}$ is the gripping force applied by the gripper's finger on the micropart $\left(F_{g}=\right.$ $\left.-F_{s}\right), L=L_{r}+L_{s}$ is the length of the complete end-effector (rigid part + force sensor), $m_{s}$ is the mass of the force sensor and $y_{C}$ is the displacement of the force sensor tip C.

Replacing (6) in (2), the following can be deduced:

$$
\left\{\begin{array}{c}
y_{A}(s)=\left[d_{p} U-H_{s}(U)-\left(1+\frac{3 L}{2 L_{a}}\right) s_{p} F_{g}-m_{s} s_{p} s^{2} y_{C}\right] D(s) \\
\alpha_{A}(s)=\left[\frac{2}{L_{a}}\left(d_{p} U-H_{s}(U)\right)-\left(\frac{3}{2 L_{a}}+\frac{3 L}{L_{a}^{2}}\right) s_{p} F_{g}-\frac{3}{2 L_{a}} m_{s} s_{p} s^{2} y_{C}\right] D(s)
\end{array}\right.
$$


The displacement at point $\mathrm{D}$ is given as follows:

$$
y_{D}=y_{A}+L \sin \alpha_{A}
$$

As the force sensor is modeled as a spring-mass-damper system, the force measured by the force sensor, $F_{S}$, is given as follows:

$$
F_{s}=-F_{g}=m_{s} \ddot{y}+b_{s}\left(\dot{y}-\dot{y}_{D}\right)+k_{s}\left(y-y_{D}\right)
$$

where $m_{s}, b_{s}$ and $k_{s}$ are respectively the mass, damping and stiffness of the force sensor; $y=y_{C}$ is the current location of the force sensor and of the micropart when the two are in contact and $\delta=y-y_{D}$ is the displacement of the force sensor due to the applied force on the force sensor $\left(F_{S}=-F_{g}\right)$.

Using (7), (8) and (9), the model of the whole smart finger is given as follows:

$$
Y=G_{u}(s)\left[d_{p} U-H_{s}-G_{f}(s) F_{g}\right]
$$

where $Y=Y(s)$ is the Laplace transform of $y$, and $G_{u}(s)$ and $G_{f}(s)$ are given by the following:

$$
\left\{\begin{array}{c}
G_{u}(s)=\frac{\left(1+\frac{2 L}{L_{a}}\right)\left(b_{s} s+k_{s}\right)}{a m_{s} s^{4}+c_{1} s^{3}+c_{2} s^{2}+\left(b_{s}+b k_{s}\right) s+k_{s}} \\
G_{f}(s)=\frac{a s^{2}+b s+1+\left(1+\frac{3 L}{L_{a}}+\frac{3 L^{2}}{L_{a}^{2}}\right) s_{p}\left(b_{s} s+k_{s}\right)}{\left(1+\frac{2 L}{L_{a}}\right)\left(b_{s} s+k_{s}\right)} \\
c_{1}=b m_{s}+a b_{s}\left(1+\frac{3 L}{2 L_{a}}\right) b_{s} m_{s} s_{p} \\
c_{2}=a k_{s}+b b_{s} m_{s}+\left(1+\frac{3 L}{2 L_{a}}\right) k_{s} m_{s} s_{p}
\end{array}\right.
$$

\section{Impedance Control with Force Tracking for the Control of the grasping Task}

In this section, a force tracking impedance control scheme is presented to control not only the interaction between the gripper's and the micropart but also the grasping force of the micropart. First, the sliding mode impedance control scheme is presented, then the force tracking problem is discussed.

\subsection{Sliding Mode Impedance Control Formulation}

The impedance control formulation is given as follows:

$$
M_{d}\left(\ddot{y}-\ddot{y}_{r}\right)+B_{d}\left(\dot{y}-\dot{y}_{r}\right)+K_{d}\left(y-y_{r}\right)=F_{g}-F_{r}
$$

where $M_{d}, B_{d}$ and $K_{d}$ are respectively the desired mass, damper and stiffness to set the desired dynamics of the contact; $y_{r}$ is the reference position to set for the position control. The sliding mode control (SMC), presented by [16], has shown its effectiveness 
for controlling nonlinear systems and for impedance control by forcing the system to slide along the sliding surface which is chosen using the impedance formulation [17] despite the robot dynamics.

The impedance error or what is also called the measure of impedance error is given in the following:

$$
e_{i}=M_{d}\left(\ddot{y}-\ddot{y}_{r}\right)+B_{d}\left(\dot{y}-\dot{y}_{r}\right)+K_{d}\left(y-y_{r}\right)+e_{f}
$$

where $e_{i}$ is the impedance error and $e_{f}=F_{r}-F_{g}$ is the force error between the desired and the applied force. $e_{i}$ and $e_{f}$ have the unit of force.

A sliding surface is chosen in [17] using the impedance control formulation defined in (12). The sliding surface is given by:

$$
\sigma=\left(\dot{y}-\dot{y}_{r}\right)+\frac{B_{d}}{M_{d}}\left(y-y_{r}\right)+\frac{K_{d}}{M_{d}} \int\left(y-y_{r}\right) d t+\frac{1}{M_{d}} \int\left(f_{r}-F_{g}\right) d t
$$

when the system reaches the sliding surface and is in sliding mode, $\sigma=0$ and $\dot{\sigma}=0$. In this case, using (13), the following is deduced:

$$
e_{i}=M_{d} \dot{\sigma}
$$

The equivalent control $u_{e q}$ is the solution of $\dot{\sigma}=0$, then replacing (10) in the derivative of (14), the following could be deduced in the frequency domain:

$$
U_{e q}(s)=\frac{Y_{r}-G_{i} F_{r}+\left(G_{f}+G_{i}\right) F_{g}}{d_{p} G_{u}}+\frac{H_{s}}{d_{p}}
$$

where $G_{i}=G_{i}(s)=\frac{1}{M_{d} s^{2}+B_{d} s+K_{d}}$ is the transfer function which represents the desired impedance formulation given in (12). The equivalent control takes effect in the sliding phase when the impedance trajectory reaches the sliding surface and is kept on it. If the system does not lie on the sliding surface, a discontinuous control is added to the equivalent control to determine the overall control action:

$$
u=u_{e q}-K \operatorname{sgn}(\sigma)
$$

where $K$ is a gain and sgn is the sign function. In many applications, the sign function causes oscillations, then the sign function could be replaced by the saturation function or others. Then the overall control action is given by the following:

$$
u=u_{e q}-\operatorname{Ksat}\left(\frac{\sigma}{\varepsilon}\right)
$$

Where $\varepsilon$ is a boundary which is a threshold chosen to reduce the oscillations and the saturation function is given as follows:

$$
\operatorname{sat}\left(\frac{\sigma}{\varepsilon}\right)=\left\{\begin{array}{lll}
\operatorname{sgn}\left(\frac{\sigma}{\varepsilon}\right) & \text { if } & |\sigma|>\varepsilon \\
\frac{\sigma}{\varepsilon} & \text { if } & |\sigma| \leq \varepsilon
\end{array}\right.
$$




\subsection{Force Tracking}

The impedance control was proposed to control the interaction between the gripper and the component. However, one weak point of the impedance control is the lack of direct force control capability. The steady state force error in the impedance control scheme is given according to [15] by:

$$
e_{f}^{s s}=k_{e q}\left[\frac{f_{r}}{k_{p}}+y_{p}-y_{r}\right]
$$

where $k_{e q}=\frac{K_{d} k_{p}}{K_{d}+k_{p}}$.

In order to cancel the steady state force error, the position reference, $y_{r}$, could be chosen as follows:

$$
y_{r}=y_{p}+\frac{f_{r}}{k_{p}}
$$

In practice, it is difficult to determine exactly the position and the stiffness of the micropart and the passive gripper. The location of the micropart and the passive gripper could be estimated using the information given by the force sensor when the measured force is different from zero. The stiffness of the environment could be estimated using the static part of (1) as follows:

$$
\hat{k}_{p}=\frac{F_{g}}{y-\hat{y}_{p}}
$$

where $\hat{y}_{p}$ is the estimated location of the micropart. However, in this paper, the passive finger's stiffness is known and so there is no need to estimate the stiffness, but the $y_{p}$ is estimated online by $\hat{y}_{p}$.

Replacing (21) in (16), the final equivalent control action is given as follows:

$$
U_{e q}(s)=\frac{\left(\frac{1}{k_{p}}-G_{i}\right) F_{r}+\left(G_{f}+G_{i}\right) F_{g}+\widehat{Y}_{p}}{d_{p} G_{u}}+\frac{H_{s}}{d_{p}}
$$

The final control action remains as in (18) with replacing $U_{e q}$ by the one defined in (23). The complete impedance control scheme is given in Fig. 4 where the SMC is the sliding mode control.

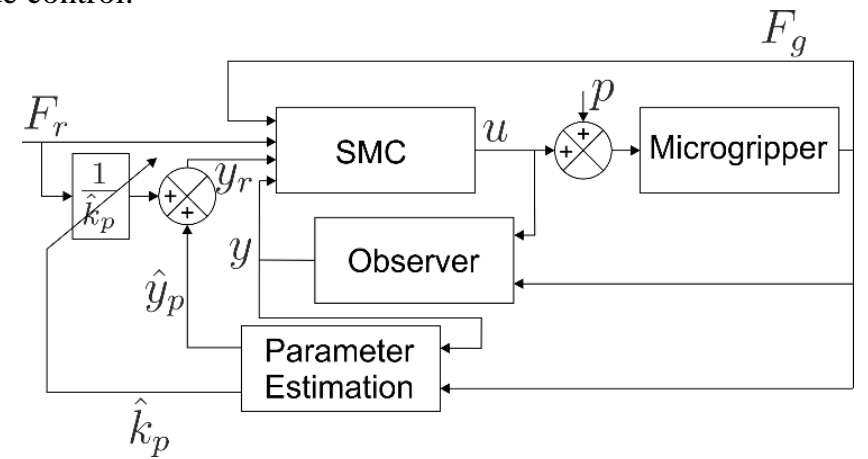

Fig. 4. Force Tracking Impedance Control Scheme. 


\section{$5 \quad$ Experimental Studies}

\subsection{Experimental Setup}

The experimental setup is composed of a two fingers microgripper. The first finger, called in this paper smart finger, is a $2 \mathrm{DoF}$ piezoelectric actuator presented in [4] where a piezoresistive force sensor is fixed at the actuator's tip. The actuator resolution is nanometric and it has a very fast response time. The force sensor design, fabrication process and performances are presented in [19]. The sensor's resolution is $100 \mathrm{nN}$ and its sensing range is $2 \mathrm{mN}$ and its natural frequency is $8 \mathrm{kHz}$. The second finger is passive, i.e. not actuated, and is made of Nickel.

A Keyence laser sensor measures the displacement of the actuator's tip, $y_{A}$. It is used to test the precision on the estimation of the position of the tip C, $y$. A microcomponent with dimensions $2 \mathrm{~mm} \times 350 \mu \mathrm{m} \times 50 \mu \mathrm{m}$ is used to test the developed grasping technique.

The microcomponent is initially manually placed onto a moving substrate which consists of a moving stage P-611.3 NanoCube from Physik Instrumente with 100 $\mu \mathrm{m}$ range and $1 \mathrm{~nm}$ in resolution and a rotation stage SR-3610-S from SmarAct with $1.1 \mu^{\circ}$ in resolution. These two devices are used to position the microcomponent between the two fingers of the microgripper in order to handle it by the microgripper.

The whole system is controlled through dSpace1104 Board with a sampling frequency of $20 \mathrm{kHz}$. The complete setup used in this paper is presented in Fig. 5.

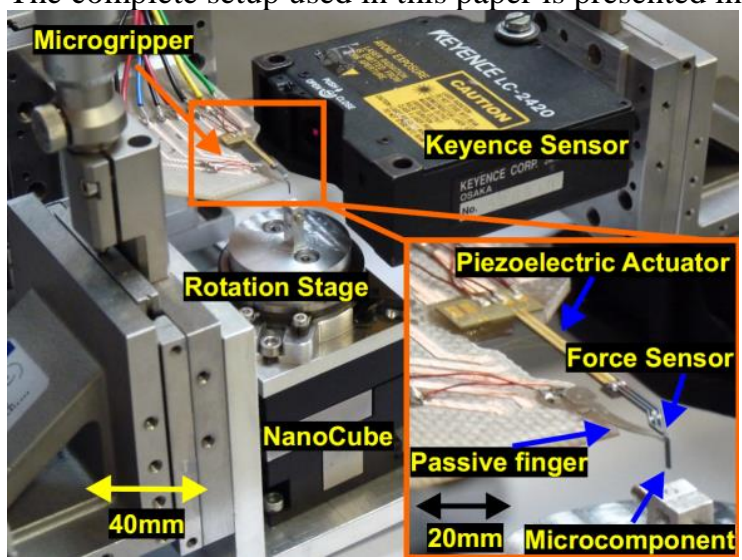

Fig. 5. The whole experimental setup including a microgripper holding a microcomponent, the nanopositioning and rotation stages and the laser sensor.

\subsection{Experimental Results}

The developed model of the complete microgripper has been validated and the method to experimentally identify the model parameters has been presented in previous works [20]. Thus, in this section the control presented in Section IV is directly tested for the automation of the grasp/release of a micropart without validating the model of the microgripper.

The strategy of manipulation is defined as follows:

1) the micropart is initially manually placed onto a substrate, 
2) the micropart is positioned close to the passive finger by moving the substrate to a small distance from it,

3) the control action starts and the smart finger approaches the micropart, enters in contact with the micropart, pushes the micropart to the passive finger until the smart finger and the micropart enter in contact with the passive finger and then the controller sets the force to the desired force,

4) the microgripper is maintained for a long period in order to test the effectiveness of the control technique in the presence of some perturbations and in the presence of the creep of the piezoelectric actuator.

Fig. 6 shows the experimental results for an automated grasp/release task of a micropart using the developed control and microgripper. The values used for the impedance are $\mathrm{Md}=1, \mathrm{Bd}=200$ and $\mathrm{Kd}=10000$.

(a)

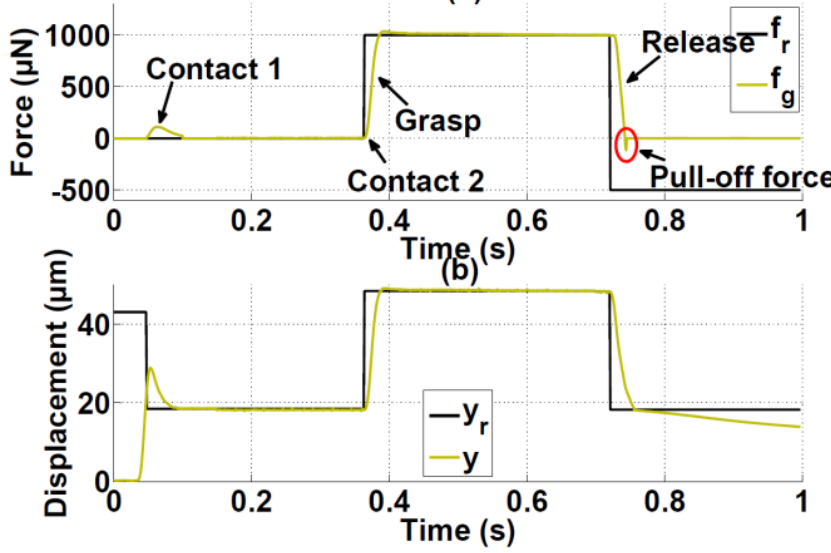

Fig. 6. The force and displacement responses of the system for the handling of the microcomponent: (a) Force response and (b) Displacement response.

First, the position of the micropart is unknown between the fingers of the microgripper. In order to be able to handle the micropart, the initial estimate of $y_{p}, \hat{y}_{p}^{0}$, is set to be big enough, $100 \mu \mathrm{m}$, to be sure that the smart finger is able to touch the micropart. Then, the controller is turned ON at time $t=50 \mathrm{~ms}$ with no force reference, the TSFM fingers start to move to let the position tracks the position reference $y_{r}$ which is equal to $\hat{y}_{p}^{0}$ because no force reference exists. Because $\hat{y}_{p}^{0}$ is bigger than the real value of $y_{-} p$, the TSFM fingers enter in contact 1 with the micropart. When contact 1 appears, a new estimation of the environment location $\hat{y}_{\mathrm{p}}$ is calculated and the position reference $y_{r}$ changes to the new estimated value $\hat{\mathrm{y}}_{\mathrm{p}}\left(y_{r}=\hat{\mathrm{y}}_{\mathrm{p}}\right)$. Then at time $t=0.37 \mathrm{~s}$, a force reference is set to the controller and contact 2 happens enabling the estimation of $\hat{\mathrm{k}}_{\mathrm{p}}$. The system reaches the steady state within a settling time of $20 \mathrm{~ms}$ and a small overshoot of $3.7 \%$ despite the online estimation of the stiffness of the micropart and the second finger. The steady state force error is null showing the significance of the parameter estimation technique and the SMIC. The automated grasping task is then achieved with success. 
The release task is then tested. For that, a negative force reference is set to the system allowing the controller to break the contact between the TSFM and the micropart and succeeding the release task within a settling time of $19 \mathrm{~ms}$. The advantage of this SMIC controller is that it can deal with pull-off force by simply applying a negative force reference to the system.

The experimental results show the significance of the approach. Comparing this approach to [24], [15], the dynamic performances are much better. Indeed, the overshoot is 10 times smaller and the response time is much smaller. Comparing the results to [18], the steady state force error is null in our work where it is bigger than $7 \%$ in the other work.

\section{Conclusion}

In this paper, a new microgripper design is proposed and tested for the automated manipulation of microcomponents. The microgripper is composed of two fingers: the first finger, called in this paper smart finger, is a piezoelectric actuator where a novel piezoresistive force sensor is fixed at the actuator's tip and the second finger is passive. A micropart of size $50 \mu \mathrm{m} \times 350 \mu \mathrm{m} \times 2 \mathrm{~mm}$ is grasped and released automatically based on a sliding mode impedance control with force tracking scheme. The control used is based on a precise dynamic nonlinear model of the microgripper.

The control approach enables setting a desired dynamic behavior to the system and the controller forces the system to follow the desired impedance and to track the desired force. This is important at the microscale to manipulate microparts without losing them. Moreover, a method to deal with pull-off force has been also considered in this paper. The automation of grasp/release task of a micropart has been done with settling time of $20 \mathrm{~ms}$ and small overshoot (less than $3.7 \%$ ). The experimental results are promising for the automation of the microassembly and micromanipulation while controlling the interaction between the microgripper and the manipulated microparts.

The proposed control approach can be extended to automate more complex microassembly and micromanipulation tasks.

\section{Acknowledgment}

These works have been funded by the Labex ACTION project (contract "ANR-11LABEX-0001-01"), ANR COLAMIR (contract " ANR-16-CE10-0009") and by the French RENATECH network through its FEMTO-ST technological facility.

\section{References}

1. Dechev, N., Cleghorn, W., Mills, J.: Microassembly of 3-d microstructurescusing a compliant, passive microgripper. J. of Microelec. Syst. 13, 176189 (2004). 
2. Bargiel, S., Rabenorosoa, K., Clévy, C., Gorecki, C., Lutz, P.: Towards micro-assembly of hybrid moems components on a reconfigurable silicon free-space micro-optical bench. J. Micromech. Microeng. 20 (2010).

3. Clévy, C., Rakotondrabe, M., Chaillet, N.: Signal Measurement and Estimation Techniques for Microand Nanotechnology. Springer (2011).

4. de Lit, P., Agnus, J., Clévy, C., Chaillet, N.: A four-degree-of freedom microprehensile microrobot on chip. Assembly and Automation 24(1), 33-42 (2004).

5. Beyeler, F., Neild, A., Oberti, S., Bell, Y. S. D.J., Dual, J., Nelson, B.: Monolithically fabricated microgripper with integrated force sensor for manipulating microobjects and biological cells aligned in an ultrasonic field. J. of Microelectromech. Systems 16(1), 7-15(2007).

6. Kim, K., Liu, X., Zhang, Y., Sun, Y.: Nanonewton force-controlled manipulation of biological cells using a monolithic mems microgripper with two-axis force feedback. J. Micromech. Microeng. 18(5), 055013 (2008).

7. Piriyanont, B., Fowler, A., Moheimani, S.: Force-controlled mems rotary microgripper. J. of Microelectromech. Syst., 24(4), 1164-1172 (2015).

8. Duc, T., Lau, G., Creemer, J., Sarro, P.: Electrothermal microgripper with large jaw displacement and integrated force sensors. J. of Microelectromech. Syst. 17(6), 1546,1555 (2008).

9. Rakotondrabe, M., Ivan, A.: Development and force/position control of a new hybrid thermo-piezoelectric microgripper dedicated to micromanipulation tasks. IEEE Trans. on Automation Science and Engineering 8(4), 824-834 (2011).

10. Wang, D., Yang, Q., Dong, H.: A monolithic compliant piezoelectric-driven microgripper: Design, modeling, and testing. IEEE/ASME Trans. on Mechatronics 18(1), 138-147 (2013).

11. Komati, B., Rabenorosoa, K., Clévy, C., Lutz, P. : Automated guiding task of a flexible micropart using a two-sensing-finger microgripper. IEEE Trans. on Automation, Science and Engineering 10(3), 515-524 (2013).

12. Hogan, N.: Impedance control - an approach to manipulation. i -theory. ii - implementation. iii - applications. ASME Transactions Journal of Dynamic Systems and Measurement Control B 107, 124 (1985).

13. Komati, B., Pac, M., Ranatunga, I., Clévy, C., Popa, D., Lutz, P. : Explicit force control v.s. impedance control for micromanipulation. In : ASME International Design Engineering Technical Conferences \& Computers and Information in Engineering Conference (IDETC) 1, Portland, USA, (2013).

14. Xie, Y., Sun, D., Tse, H. Y. G., Liu, C., Cheng, S. H.: Force sensing and manipulation strategy in robot-assisted microinjection on zebrafish embryos. IEEE/ASME Transactions on Mechatronics 16(6), 1002-1010 (2011).

15. Seraji, H., Colbaugh, R.: Force tracking in impedance control. The Int. J. of Robotics Research 16(1), 97-117 (1997).

16. Utkin, V.: Variable structure systems with sliding modes. IEEE Trans. on Automatic Control, 22 (2), 212-222 (1977).

17. Lu, Z., Goldenberg, A.: Robust impedance control and force regulation: Theory and experiments. The Int. J. of Robotics Research 14(3), 225-254 (1995).

18. Xu, Q.: Precision position/force interaction control of a piezoelectric multimorph microgripper for microassembly. IEEE Trans. on Automation, Science and Engineering 10(3), $503-$ 514 (2013).

19. Komati, B., Agnus, J., Clévy, C., Lutz, P.: Prototyping of a highly performant and integrated piezoresistive force sensor for microscale applications. J. Micromech. Microeng. 24(3), 035018 (2014). 
20. Komati, B., Clévy, C., Rakotondrabe, M., Lutz, P. : Dynamic force/position modeling of a one-dof smart piezoelectric micro-finger with sensorized end-effector. In: IEEE/ASME Int. Conf. on Advanced Intelligent Mechatronics, 1474-1479, Besançon (2014).

21. Ballas, R.: Piezoelectric Multilayer Beam Bending Actuators: Static and Dynamic Behavior and Aspects of Sensor Integration. Springer (2007).

22. Low, T., Guo, W.: Modeling of a three-layer piezoelectric bimorph beam with hysteresis. J. Microelectromech. Syst. 4(4), 230237 (1995).

23. Rakotondrabe, M., Haddab, Y. and Lutz, P.: Quadrilateral modelling and robust control of a nonlinear piezoelectric cantilever. IEEE Trans. on Control Systems Technology 17, 528539 (2009).

24. Komati, B., Clévy, C., Lutz, P.: Force tracking impedance control with unknown environment at the microscale. In: IEEE ICRA Int. Conf. on Robotics and Automation, Hong Kong, China (2014). 